\title{
Strongly Annular Functions with Small Taylor Coefficients
}

\author{
Daniel D. Bonar ${ }^{1}$, Frank Carroll ${ }^{2}$, and George Piranian ${ }^{3}$ \\ ${ }^{1}$ Department of Mathematical Sciences, Denison University, Granville, OH 43023, USA \\ ${ }^{2}$ Department of Mathematics, Ohio State University, Columbus, OH 43210, USA \\ ${ }^{3}$ Department of Mathematics, University of Michigan, Ann Arbor, MI 48104, USA
}

\section{Introduction}

A holomorphic function $f$ in the unit disk $D$ is strongly annular provided there exists a sequence $\left\{r_{m}\right\}$ such that $r_{m} \nearrow 1$ and

$$
\lim _{m \rightarrow \infty} \min _{|z|=r_{m}}|f(z)|=\infty \text {. }
$$

The simplest examples of strongly annular functions have the form

$$
f(z)=\sum_{m=1}^{\infty} c_{m} z^{k_{m}}
$$

where the sequence of coefficients $c_{m}$ increases fast enough so that

$$
\left|c_{1}\right|+\cdots+\left|c_{m-1}\right|=o\left(c_{m}\right)
$$

and where the sequence of exponents $k_{m}$ increases so rapidly that on the circle $|z|=1$ $-1 / k_{m}$ the $m^{\text {th }}$ term of the series in (1) is much larger than the sum of the later terms. (See [4, Chapter 2, Section 13].)

It is natural to ask whether unboundedness of the sequence of Taylor coefficients is necessary for strong annularity, and whether the Taylor series of a strongly annular function must be almost lacunary in an appropriate sense (see [1, p. 59, Question 6.9]).

The answer to both questions is negative. For instance, Howell [3] has recently shown that if we impose the topology of uniform convergence on compact subsets of $D$ on the space of functions $\sum_{0}^{\infty}( \pm 1) z^{n}$, then the strongly annular functions in that space constitute a residual subset of the space.

We shall exhibit an explicit counterexample for both questions. Our auxiliary computations yield new information about the Taylor coefficients of the $k^{\text {th }}$ power $b^{k}$ of a univalent function $b$ from $D$ onto $D$. 
If in (1) we replace $z$ with its Möbius transform

$$
b(z)=\frac{1 / 2-z}{1-z / 2}
$$

our function $f$ takes the form

$$
f(z)=\sum_{m=1}^{\infty} c_{m}[b(z)]^{k_{m}}=\sum_{n=0}^{\infty} a_{n} z^{n} .
$$

We shall use the interchangeable symbols $\alpha(k, n)$ and $\alpha_{k n}$ to denote the $n^{\text {th }}$ Taylor coefficient (at the origin) of the function $[b(z)]^{k}$. With this notation, we can write the Taylor coefficients of our new function $f$ in the form

$$
a_{n}=\sum_{m=1}^{\infty} c_{m} \alpha\left(k_{m}, n\right)
$$

In Section 2, we analyze the sequences $\left\{\alpha_{k n}\right\}_{n=0}^{\infty}(k=1,2, \ldots)$. We find that, with the notation

$$
A(k)=\max _{n}\left|\alpha_{k n}\right|
$$

the order of magnitude of $A(k)$ is $k^{-1 / 3}$. The coefficients $\alpha_{k n}$ whose order of magnitude is $k^{-1 / 3}$ occur in long blocks, and their indices $n$ have the order of magnitude $k$.

In Section 3, we choose the sequences $\left\{c_{m}\right\}$ and $\left\{k_{m}\right\}$ that produce our example. Indeed, if $c_{m}=k_{m}^{1 / 3} m^{-1 / 2}$ and $k_{m} \rightarrow \infty$ fast enough, then $a_{n} \rightarrow 0$, but $a_{n}=\Omega\left(m^{-1 / 2}\right)$ for many indices $n$ near $3 k_{m}$. It follows that $f$ is not the sum of a bounded function and a function whose Taylor series at the origin is even mildly lacunary.

\section{Bounds on the Taylor Coefficients}

Theorem 1. There exist positive numbers $A_{1}$ and $A_{2}$ such that for all $k$ and $n$ the coefficients $\alpha_{k n}$ in (2) satisfy the inequality

$$
\left|\alpha_{k n}\right|<A_{1} k^{-1 / 3}
$$

and such that, for each nonnegative integer $j$,

$$
\liminf _{k \rightarrow \infty} k^{1 / 3}|\alpha(k, 3 k+j)|>A_{2} .
$$

In addition to estimates on the largest coefficients in the Taylor series for $b^{k}$, we need upper bounds on the coefficients outside of certain blocks.

Theorem 2. The coefficients $\alpha_{k n}$ in (2) satisfy the inequalities

$$
\begin{array}{ll}
\left|\alpha_{k n}\right| \leqq 6 /(k-3 n) \pi & (n<k / 3) \\
\left|\alpha_{k n}\right| \leqq 2 /(n-3 k) \pi & (n>3 k) .
\end{array}
$$


We devote the remainder of this section to the proofs of Theorems 1 and 2, and we suggest that readers who wish to avoid computations proceed to Section 3 , where we apply the theorems to the construction of our example.

It is convenient to begin with the proof of Theorem 2. By Cauchy's formula,

$$
\alpha_{k n}=\frac{1}{2 \pi} \int_{-\pi}^{\pi}\left[b\left(e^{i \theta}\right)\right]^{k} e^{-i n \theta} d \theta
$$

To put the integrand into manageable form, we observe that because $\left|b\left(e^{i \theta}\right)\right|=1$ and $b(-1)=1$, we may write $b\left(e^{i \theta}\right)=\exp [i \psi(\theta)]$, where $\psi$ is a continuously differentiable function and $\psi(-\pi)=0$. Since

$$
b^{\prime}(z)=\frac{-3 / 4}{(1-z / 2)^{2}} \quad \text { and } \quad \psi^{\prime}(\theta)=\left|b^{\prime}\left(e^{i \theta}\right)\right| \text {, }
$$

we see that

$$
\psi^{\prime}(\theta)=\frac{3}{4\left|1-e^{i \theta} / 2\right|^{2}}=\frac{3}{5-4 \cos \theta} .
$$

Because the coefficients $\alpha_{k n}$ are real, Cauchy's formula implies that

$$
\alpha_{k n}=\frac{1}{2 \pi} \int_{-\pi}^{\pi} \cos [k \psi(\theta)-n \theta] d \theta .
$$

Letting $g(\theta)$ denote the expression in brackets in the integrand, and observing that $g(-\pi)=n \pi$, we now have the formulas

$$
\alpha_{k n}=\frac{1}{2 \pi} \int_{-\pi}^{\pi} \cos g(\theta) d \theta
$$

and

$$
\begin{aligned}
& g(\theta)=\int_{-\pi}^{\theta}\left(\frac{3 k}{5-4 \cos t}-n\right) d t+n \pi, \\
& g^{\prime}(\theta)=\frac{3 k}{5-4 \cos \theta}-n, \\
& g^{\prime \prime}(\theta)=\frac{-12 k \sin \theta}{(5-4 \cos \theta)^{2}} .
\end{aligned}
$$

Since $g^{\prime \prime}(\theta)$ and $\sin \theta$ have opposite signs, the maximum and minimum values of $g^{\prime}$ on $[-\pi, \pi]$ are

$$
g^{\prime}(0)=3 k-n \quad \text { and } \quad g^{\prime}(\pi)=g^{\prime}(-\pi)=\frac{k}{3}-n .
$$

We note that $g^{\prime}$ is an even function and that it decreases in $[0, \pi]$.

To obtain the bounds (5) and (6), we shall use the following proposition from calculus. 
Lemma. Suppose that $g$ is a real-valued function on the interval $[a, b]$, that $\mathrm{g}^{\prime}$ exists and is monotone on $[a, b]$, and that $\left|g^{\prime}(x)\right| \geqq B>0$ on $[a, b]$. Then

$$
\left|\int_{a}^{b} \cos g(x) d x\right| \leqq 2 / B .
$$

In proving the lemma, we can obviously restrict ourselves to the case where $g^{\prime}$ is increasing and $g^{\prime}(a) \geqq B$. Making the change of variable $t=g(x)$ and applying Bonnet's form of the second mean-value theorem [2, p. 311], we obtain the relations

$$
\int_{a}^{b} \cos g(x) d x=\int_{\alpha}^{\beta} \frac{\cos t d t}{g^{\prime}(x(t))}=\frac{1}{g^{\prime}(a)} \int_{\alpha}^{\xi} \cos t d t,
$$

where $\xi$ denotes an appropriate point between $\alpha$ and $\beta$. The lemma now follows immediately.

To establish the bound (5) in Theorem 2, we apply the lemma to our function $g$ on the intervals $[-\pi, 0]$ and $[0, \pi]$, with $B=k / 3-n$. To establish $(6)$, we proceed similarly, using the value $B=n-3 k$. This completes the proof of Theorem 2 .

In the proof of Theorem 2, we were able to use our lemma because for $n<k / 3$ and $n>3 k$, the derivative $g^{\prime}$ has no zeros on the interval $[-\pi, \pi]$. In the proof of Theorem 1, we may have to cope with one or two zeros of $g^{\prime}$. Moreover, even if $g^{\prime}$ has no zeros, the minimum value of $\left|g^{\prime}(\theta)\right|$ may be so small that the lemma does not give the inequality (3), which for some values of $n$ is much stronger than (5) and (6).

Let $\theta_{0}$ denote the point in $[0, \pi]$ where $\left|g^{\prime}(\theta)\right|$ takes its minimum value. Clearly, the contribution from the interval $\left[\theta_{0}-k^{-1 / 3}, \theta_{0}+k^{-1 / 3}\right]$ to the integral in (7) is less than $2 k^{-1 / 3}$.

In the set $[0, \pi] \backslash\left[\theta_{0}-k^{-1 / 3}, \theta_{0}+k^{-1 / 3}\right]$, the value of $\left|g^{\prime}(\theta)\right|$ is

$$
\left|g^{\prime}\left(\theta_{0}\right)\right|+\left|\int_{\theta_{0}}^{\theta} g^{\prime \prime}(t) d t\right| .
$$

By virtue of the third formula in (8), the second term in this expression is greater than

$$
\left|\int_{\theta_{0}}^{\theta_{0} \pm k^{-1 / 3}} \frac{12 k \sin t d t}{81}\right|
$$

where the sign is chosen so that the point $\theta_{0} \pm k^{-1 / 3}$ lies in $[0, \pi]$. Thus, with an obvious choice of the ambiguous sign, we obtain for all $\theta$ in the interval the inequalities

$$
\begin{aligned}
\left|g^{\prime}(\theta)\right| & >\left|\int_{\theta_{0}}^{\theta_{0} \pm k^{-1 / 3}} g^{\prime \prime}(t) d t\right| \geqq\left|\int_{\theta_{0}}^{\theta_{0} \pm k^{-1 / 3}} \frac{12 k \sin t d t}{81}\right| \\
& \geqq \int_{0}^{k^{-1 / 3}} \frac{12 k}{81} \frac{2 t}{\pi} d t=4 k^{1 / 3} / 27 \pi .
\end{aligned}
$$

Applying the lemma and using similar considerations for the interval $[-\pi, 0]$, we arrive at the estimate (3). 
The proof of the second part of Theorem 1 is more delicate. Before giving its details, we point out that if $g^{\prime}(\theta)$ is fairly small at a point where $g^{\prime \prime}(\theta)=0$, then near that point the integrand in (7) changes so slowly that $\alpha_{k n}$ may be relatively large.

We let $j$ denote a nonnegative integer, and we consider the coefficient $\alpha(k, n)$ $=\alpha(k, 3 k+j)$, where $k$ is much larger than $j$. It is convenient to introduce the function $h$ defined by the equation

$$
g(\theta)=k \pi-h(\theta) .
$$

We can easily verify that $h(0)=0$; since $h^{\prime}$ is an even function, it follows that $h$ is odd. By virtue of the second formula in (8), we can write

$$
h^{\prime}(\theta)=j+3 k\left[1-\frac{1}{1+4(1-\cos \theta)}\right] \text {. }
$$

Let $\theta_{1}$ and $\theta_{2}$ denote the first two points in $[0, \pi]$ where $\cos h(\theta)=0$. Then

$$
\int_{0}^{\pi} \cos h(\theta) d \theta=\left(\int_{0}^{\theta_{1}}+\int_{\theta_{1}}^{\theta_{2}}+\int_{\theta_{2}}^{\pi}\right) \cosh (\theta) d \theta .
$$

Because $h^{\prime}$ is an increasing function in $[0, \pi]$, the first integral on the right is greater than $2 \theta_{1} / \pi$. The second integral is negative, and its absolute value is less than $\theta_{2}-\theta_{1}$. With the change of variable $t=h(\theta)$, the third integral takes the form

$$
\int_{h\left(\theta_{2}\right)}^{h(\pi)} \frac{\cos t d t}{h^{\prime}(\theta(t))}
$$

Because the denominator in the integrand is an increasing function of $t$, we can regard this integral as a finite series with decreasing terms of alternate signs, the first term being positive. In other words, the third integral is positive; we shall ignore it.

To obtain a positive lower bound on the sum of the first two of our integrals, we need estimates on $\theta_{1}$ and $\theta_{2}$. By (9), the function $h^{\prime}$ has a Taylor series

$$
h^{\prime}(\theta)=j+3 k\left(2 \theta^{2}+\cdots\right)
$$

and since $h(0)=0$, it follows that

$$
h(\theta)=j \theta+2 k\left(\theta^{3}+\cdots\right) \text {. }
$$

Consequently, in the particular case where $j=0$,

$$
\begin{aligned}
& \theta_{1}=(\pi / 4 k)^{1 / 3}[1+o(1)], \\
& \theta_{2}=3^{1 / 3} \theta_{1}[1+o(1)] .
\end{aligned}
$$

Moreover, these formulas hold also for positive values of $j$, provided $j=o\left(k^{1 / 3}\right)$. Therefore

$$
|\alpha(k, 3 k+j)|>\frac{1}{2 \pi}\left[\frac{2 \theta_{1}}{\pi}-\left(\theta_{2}-\theta_{1}\right)\right] \sim \frac{\theta_{1}}{2 \pi}\left[\frac{2}{\pi}-\left(3^{1 / 3}-1\right)\right] .
$$

Because the last expression in brackets is positive and $\theta_{1} \sim(\pi / 4 k)^{1 / 3}$, this establishes the relation (4), and the proof of Theorem 1 is complete. 
Remarks. 1. We have shown that the Taylor series of $b^{k}$ has a long block of relatively large coefficients with index near $3 k$. Obviously, a similar block occurs near $n=k / 3$. It is fairly easy to verify that the two blocks extend to both sides of $3 k$ and $k / 3$, respectively.

2. A careful inspection of our proof shows that we can make the second assertion in Theorem 1 slightly stronger: There exists a positive constant $\eta$ such that $|\alpha(k, 3 k+j)|>A_{2} k^{-1 / 3}$ whenever $k$ is large enough and $0 \leqq j \leqq \eta k^{1 / 3}$.

3. Theorems 1 and 2 have obvious analogues for the more general functions

$$
\left(\frac{w-z}{1-\bar{w} z}\right)^{k}=\sum_{n=0}^{\infty} \alpha(k, n, w) z^{n},
$$

where $w$ denotes any point in the unit disk $(w \neq 0)$. Naturally, some of the parameters in the analogues depend on $|w|$; but the exponents $\pm 1 / 3$ survive.

\section{The Example}

Theorem 3. If $\left\{k_{m}\right\}$ is a sequence of positive integers and $k_{m} \rightarrow \infty$ rapidly enough, then the function $f$ defined by the formula

$$
f(z)=\sum_{m=1}^{\infty} \frac{k_{m}^{1 / 3}}{m^{1 / 2}}[b(z)]^{k_{m}}=\sum_{n=0}^{\infty} a_{n} z^{n}
$$

is strongly annular and has the additional properties

(i) $\lim _{n \rightarrow \infty} a_{n}=0$,

(ii) in every decomposition $f=f_{1}+f_{2}$, where $f_{1}(z)=\sum d_{n} z^{\lambda_{n}}$ and $\lambda_{n+1}-\lambda_{n} \geqq 2$ for all $n$, the component $f_{2}$ fails to belong to the Hardy class $H^{2}$.

It is easy to choose the exponents $k_{m}$ so that $f$ is strongly annular. Indeed, on the circle $|z|=1$ the $m^{\text {th }}$ term in the series $(10)$ has modulus $k_{m}^{1 / 3} m^{-1 / 2}=B_{m}$, and therefore there exists a circle $|z|=r_{m}\left(r_{m}<1\right)$ on which the term has modulus greater than $B_{m} / 2$. If $k_{m}$ is large enough, this is greater than $4\left(B_{1}+B_{2}+\cdots+B_{m-1}\right)$. If the exponents $k_{m+1}, k_{m+2}, \ldots$ are large enough, then the sum of the corresponding terms is smaller than $B_{m} / 8$ on the circle $|z|=r_{m}$, and therefore $|f(z)|>B_{m} / 4$ whenever $|z|=r_{m}$.

To make certain that $a_{n} \rightarrow 0$, we require first that no two of the intervals $\left[k_{m} / 6,4 k_{m}\right]$ overlap. If the index $n$ does not lie in the $m^{\text {th }}$ of these intervals, then the bounds in Theorem 2 give the inequality

$$
k_{m}^{1 / 3} m^{-1 / 2}\left|\alpha_{k n}\right|<k_{m}^{1 / 3} m^{-1 / 2} \frac{1}{\pi k_{m} / 6}=\frac{6}{\pi m^{1 / 2} k^{2 / 3}} .
$$

If $n$ lies in the $m^{\text {th }}$ interval, we invoke the bound (3) and obtain the inequality

$$
k_{m}^{1 / 3} m^{-1 / 2}\left|\alpha_{k n}\right|<A_{1} m^{-1 / 2} .
$$

Now we subject the sequence $\left\{k_{m}\right\}$ to the additional requirement that $\sum k_{m}^{-2 / 3}<\infty$, 
and we observe that for each integer $k$ the sequence $\left\{\alpha_{k n}\right\}_{n=0}^{\infty}$ converges to 0 . Clearly, $f$ has property (i).

Finally, suppose that the equation $f=f_{1}+f_{2}$ represents a decomposition of $f$ such that one of every pair of consecutive coefficients in the power series of $f_{1}$ is zero. By the second part of Theorem 1, the two coefficients with index $3 k_{m}$ and $3 k_{m}$ +1 in the power series of the $m^{\text {th }}$ term in the middle member of $(10)$ have modulus greater than $A_{2} / m^{1 / 2}$. If the sequence $\left\{k_{m}\right\}$ increases fast enough, then one of the two corresponding Taylor coefficients of $f_{2}$ has modulus at least $A_{2} / 2 \mathrm{~m}^{1 / 2}$. Since $\sum 1 / \mathrm{m}$ $=\infty$, we see at once that $f_{2} \notin H^{2}$. Therefore $f$ has the property (ii), and the proof of Theorem 3 is complete.

The reader may feel that while the function (10) does not have an essentially lacunary power series, we have merely hidden the lacunarity by means of a Möbius transformation. To carry the obliteration of lacunarity a step further, we could replace each function $[b(z)]^{k_{m}}$ in (10) with the corresponding function

$$
\left(\frac{e^{m i} / 2-z}{1-z e^{-m i} / 2}\right)^{k_{m}}
$$

\section{References}

1. Bonar, D.D.: On annular functions. Berlin: VEB Deutscher Verlag der Wissenschaften 1971

2. Franklin, P.: A Treatise on Advanced Calculus. New York: Wiley 1940

3. Howell, R.: Annular functions in probability. Proc. Amer. math. Soc. 52, 217-221 (1975)

4. Priwalow, I.I.: Randeigenschaften analytischer Funktionen. Berlin: VEB Deutscher Verlag der Wissenschaften 1956 